\title{
Modelling inactivation of Staphylococcus aureus and Yersinia enterocolitica by high-pressure homogenisation at different temperatures
}

\author{
Ann M.J. Diels, Elke Y. Wuytack, Chris W. Michiels* \\ Laboratory of Food Microbiology, Katholieke Universiteit Leuven, Kasteelpark Arenberg 22, B-3001, Leuven, Belgium
}

Received 1 May 2002; received in revised form 28 October 2002; accepted 27 December 2002

\begin{abstract}
A detailed study of the inactivation of Staphylococcus aureus and Yersinia enterocolitica by high-pressure homogenisation was performed at, respectively, 25 and 35 different combinations of process temperature and process pressure covering a range of $5-50{ }^{\circ} \mathrm{C}$ and $100-300 \mathrm{MPa}$. It appeared that in the entire studied pressure-temperature domain, S. aureus was more resistant to high-pressure homogenisation than $Y$. enterocolitica. Furthermore, the effect of the process pressure on the inactivation of $S$. aureus was considerably smaller than on the inactivation of $Y$. enterocolitica. Also, temperature between 5 and $40{ }^{\circ} \mathrm{C}$ did not affect inactivation of $S$. aureus by high-pressure homogenisation, while $Y$. enterocolitica inactivation was affected by temperature over a much wider range.

Different mathematical models were compared to describe the inactivation of both bacteria under the experimental conditions applied. Such pressure-temperature inactivation models form the engineering basis for design, evaluation and optimisation of high-pressure homogenisation processes as a new preservation technique.

(C) 2003 Elsevier Science B.V. All rights reserved.
\end{abstract}

Keywords: High-pressure homogenisation; Inactivation; Staphylococcus aureus; Yersinia enterocolitica; Mathematical modelling

\section{Introduction}

Heat treatment is one of the most commonly used preservation treatments for food and other perishable products. It is an efficient and economical process to inactivate microorganisms, but it cannot be used to treat heat-labile compounds. Therefore, over the last 10 years, considerable research efforts have been

* Corresponding author. Tel.: +32-16-32-15-78; fax: +32-1632-19-60.

E-mail address: chris.michiels@agr.kuleuven.ac.be (C.W. Michiels). directed towards the development of novel nonthermal processes for preservation, such as the use of high hydrostatic pressure, pulsed electric field, ultraviolet light, pulsed light and high-pressure homogenisation.

It is known that high-pressure homogenisation can inactivate microorganisms (Popper and Knorr, 1990; Lanciotti et al., 1994, 1996; Guerzoni et al., 1999). Several studies have indicated that Gram-negative bacteria are more sensitive to high-pressure homogenisation than Gram-positive bacteria (Kelemen and Sharpe, 1979; Vachon et al., 2002; Wuytack et al., 2002). This suggests a correlation between cell wall structure and high-pressure resistance, which indicates 
that high-pressure homogenisation kills vegetative bacteria mainly through mechanical destruction of the cell integrity, caused by the spatial pressure and velocity gradients, turbulence (Doulah et al., 1975), impingement (Engler and Robinson, 1981; Keshavarz-Moore et al., 1990) and/or cavitation (Save et al., 1994; Shirgaonkar et al., 1998), which occur in a liquid during high-pressure homogenisation. Vachon et al. (2002) have made transmission electron micrographs of high-pressure homogenised cells of $L$. monocytogenes, which illustrated that cells are indeed disrupted by high-pressure homogenisation. The finding that high-pressure homogenisation does not cause sublethal injury reflects the 'all or nothing' impact of this technique on bacteria (Wuytack et al., 2002). Bacteria are either disrupted or not.

Furthermore, it has been observed that several successive rounds of high-pressure homogenisation have an additive effect on the viability reduction, which opens perspectives to increase the microbicidal efficiency of the treatment (Wuytack et al., 2002).

Up to date quantitative data to describe inactivation by high-pressure homogenisation are scarce. The objective of this study is therefore to study in more detail the effect of process parameters as pressure and temperature on the inactivation of bacteria by highpressure homogenisation. Staphylococcus aureus and Yersinia enterocolitica were chosen as reference bacteria since an earlier study indicated that these were the most resistant of, respectively, five tested Grampositive bacteria and six tested Gram-negative bacteria (Wuytack et al., 2002). A mathematical model will be proposed to quantify the impact of a highpressure homogenisation treatment on $S$. aureus and $Y$. enterocolitica as function of homogenisation pressure and temperature.

\section{Materials and methods}

\subsection{Preparation of cell suspensions}

S. aureus and Y. enterocolitica were used throughout this study. Nutrient broth (CM76 Oxoid, UK) cultures $(40 \mathrm{ml})$ in $100 \mathrm{ml}$ Erlenmeyers were grown to stationary phase by shaking (200 rpm) for $21 \mathrm{~h}$ at $30{ }^{\circ} \mathrm{C}$ (Y. enterocolitica) or at $37{ }^{\circ} \mathrm{C}(S$. aureus $)$, centrifuged at $4000 \times g$ for $5 \mathrm{~min}$, and the pellets were resuspended to a concentration of approximately $10^{8}$ $\mathrm{cfu} / \mathrm{ml}$ in sterile PBS buffer $(10 \mathrm{mM}$ potassium phosphate buffer, $\mathrm{pH} 7.0 ; 8.4 \mathrm{~g} / \mathrm{l} \mathrm{NaCl})$.

\subsection{High-pressure homogenisation treatment}

Cell suspensions were homogenised at pressures ranging between 100 and $300 \mathrm{MPa}$ and temperatures between 5 and $50{ }^{\circ} \mathrm{C}$. The used temperature-pressure combinations and the number of observations at each combination are shown in Table 1. The high-pressure homogeniser was an Emulsiflex C5 (Avestin, Ottowa, Canada) consisting of an inlet reservoir, an air-driven pump and a ceramic homogenising valve. A heat exchanger was placed immediately after the homogenising valve to withdraw the heat generated during passage of the sample through the homogenising valve. In addition, the entire homogeniser was immersed in a water bath to maintain the desired process temperature and to improve heat dissipation from the homogenising valve block. The outlet temperature of a typical sample homogenised at $300 \mathrm{MPa}$ was without heat exchanger, $\pm 20{ }^{\circ} \mathrm{C}$ higher than the process temperature but was reduced to $18{ }^{\circ} \mathrm{C}$ after passage through the heat exchanger whatever the process temperature.

\subsection{Determination of the loss of viability}

To assess loss of viability caused by a treatment, untreated and treated cell suspensions were serially diluted in PBS and plated on the surface of plate count agar (PCA, CM325 Oxoid) medium. Plates were incubated for $21 \mathrm{~h}$ at $30{ }^{\circ} \mathrm{C}$ ( . enterocolitica) or at $37{ }^{\circ} \mathrm{C}$ (S. aureus), and the number of colony forming

Table 1

Number of observations at each temperature-pressure combination for $S$. aureus $(\mathrm{S})$ and $Y$. enterocolitica $(\mathrm{Y})$

\begin{tabular}{|c|c|c|c|c|c|c|c|c|c|c|}
\hline \multirow{2}{*}{$\begin{array}{l}\text { Temperature } \\
\left({ }^{\circ} \mathrm{C}\right)\end{array}$} & \multicolumn{2}{|c|}{$100 \mathrm{MPa}$} & \multicolumn{2}{|c|}{$150 \mathrm{MPa}$} & \multicolumn{2}{|c|}{$200 \mathrm{MPa}$} & \multicolumn{2}{|c|}{$250 \mathrm{MPa}$} & \multicolumn{2}{|c|}{$300 \mathrm{MPa}$} \\
\hline & $S$ & $\mathrm{Y}$ & $\mathrm{S}$ & $\mathrm{Y}$ & $\mathrm{S}$ & $\mathrm{Y}$ & S & $\mathrm{Y}$ & $\mathrm{S}$ & Y \\
\hline 5 & 2 & 3 & 1 & 3 & 2 & 3 & 2 & 3 & 2 & 2 \\
\hline 10 & 3 & 5 & 3 & 5 & 2 & 2 & 2 & 3 & 0 & 3 \\
\hline 15 & 0 & 6 & 0 & 5 & 0 & 6 & 0 & 6 & 0 & 4 \\
\hline 20 & 0 & 3 & 0 & 3 & 0 & 3 & 0 & 3 & 0 & 2 \\
\hline 25 & 3 & 5 & 3 & 5 & 3 & 5 & 3 & 3 & 3 & 5 \\
\hline 35 & 3 & 3 & 3 & 3 & 3 & 5 & 3 & 8 & 1 & 2 \\
\hline 40 & 0 & 3 & 0 & 3 & 0 & 3 & 0 & 0 & 0 & 0 \\
\hline 45 & 4 & 3 & 4 & 2 & 4 & 0 & 4 & 0 & 1 & 0 \\
\hline 50 & 3 & 3 & 3 & 0 & 3 & 0 & 3 & 0 & 1 & 0 \\
\hline
\end{tabular}


units (CFU) was determined. The logarithm of the reduction factor (log RF) was calculated as

$\operatorname{logRF}=\log \left(\frac{\text { CFU before treatment }}{\text { CFU after treatment }}\right)$.

\subsection{Data analysis and model evaluation}

Starting from a second-order polynomial model describing $\log \mathrm{RF}$ as a function of pressure $(P)$ and temperature $(T)$, a backward regression procedure using the SAS package release 8.01 (SAS online Doc, Version 8) was applied to propose derived models and to determine the parameters of, and evaluate, these models. The backward regression procedure begins with all candidate variables in the model and then systematically removes variables that are not significantly associated $(P>0.1)$ with the target, until a model with only significant parameters is obtained.

The regression procedure calculates the set of parameters with the lowest sum of squares of errors (SSE).

$\mathrm{SSE}=\sum\left(\operatorname{logRF} \mathrm{experimental}-\operatorname{logRF_{\text {fitted}}}\right)^{2}$

A student $t$-test was done to evaluate the estimated parameters. Therefore, the $t$-value and $p$-value were determined.

$t=\frac{\text { estimated parameter }}{\text { standard error of the parameter }}$

$p$-Value is the corresponding probability for testing the null hypothesis that the parameter is not significantly different from zero.

To evaluate the proposed models, an adjusted regression coefficient $r^{2}$ and the root mean square error (RMSE) were determined. In general, $r^{2}$ measures how well a linear or a nonlinear model fit the data. Because this statistical parameter is largely dependent on the model structure and on the number of observations $(n)$ and parameters $(p)$, an adjusted $r^{2}$ was used to compare different nonlinear models on their ability to fit data sets, making allowance for the number of observations and parameters:

Adjusted $r^{2}=1-\left(\frac{(n-i)\left(1-r^{2}\right)}{(n-p)}\right)$ $i$ is an indicator variable that is 1 if the model includes an intercept, and 0 otherwise.

The RMSE measures the average deviation between the observed and the fitted values.

$\mathrm{RMSE}=\sqrt{\frac{\mathrm{SSE}}{n-p}}$

A measure of the reliability of the parameters to predict the loss of viability (log RF) caused by a highpressure homogenisation treatment was obtained by comparing the experimentally determined log RF values to the ones fitted by the model. The fits obtained for the various models were also examined for the distribution of residuals. Residuals of an appropriate fit should be distributed randomly and not systematically related to the applied pressure or temperature.

Furthermore, a statistical comparison of the different nonlinear models was made by calculating Mallows's criterion $\left(C_{p}\right)$ (Mallows, 1973), Akaike's information criterion (AIC) (Akaike, 1969), Schwarz Bayesian criterion (SBC) (Schwarz, 1978) and the predictive residual sum of squares (PRESS). These criteria, except PRESS, compare models by their sum of squares of residuals (SSE), corrected for the number of parameters and the number of observations.

$C_{p}=\left(\frac{\mathrm{SSE}_{p}}{s^{2}}\right)-(n-2 p)$

where $s^{2}$ is the MSE for the full model and $\mathrm{SSE}_{p}$ is the sum of squares of errors for a model with $p$ parameters including the intercept, if any.

$\mathrm{AIC}=n \ln \frac{\mathrm{SSE}}{n}+2 p$

$\mathrm{SBC}=n \ln \frac{\mathrm{SSE}}{n}+p \ln n$

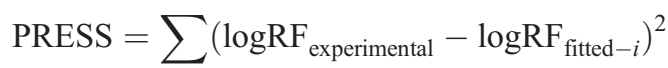

$\log \mathrm{RF}_{\text {fitted- } i}$ is the fitted value for the $i$ th observation that results from dropping the $i$ th observation from the parameter estimates.

This comparison is only valid when the errors are normally distributed. The model with the lowest $C_{p}$, 
AIC, SBC and PRESS is the best choice from a statistical point of view.

Based on log RF values calculated with the selected models, pressure-temperature diagrams were drawn using Microsoft Excel to show the pressuretemperature combinations resulting in the same inactivation $(\log R F)$.

\section{Results and discussion}

\subsection{Inactivation of $Y$. enterocolitica and S. aureus by high-pressure homogenisation}

The inactivation of $Y$. enterocolitica and $S$. aureus caused by high-pressure homogenisation treatments $\left(100-300 \mathrm{MPa}\right.$ and $\left.5-50{ }^{\circ} \mathrm{C}\right)$ is represented, respectively, in Fig. 1A and B. It appears that in the entire studied pressure-temperature domain, $S$. aureus is more resistant to high-pressure homogenisation than $Y$. enterocolitica. High-pressure homogenisation caused almost no inactivation of $S$. aureus at $35^{\circ} \mathrm{C}$ and below, even up to a process pressure of $300 \mathrm{MPa}$. However, above a process temperature of $35{ }^{\circ} \mathrm{C}$, the inactivation degree abruptly increased for high-pressure homogenisation treatments with a process pressure higher than $100 \mathrm{MPa}$. A homogenisation pressure of $100 \mathrm{MPa}$ caused already $1 \log$ unit inactivation of $Y$. enterocolitica even at low temperature. An increase in the process pressure resulted in a gradual increase of inactivation, which is more pronounced when the process temperature is higher.

From Fig. 1, it can also be concluded that for $S$. aureus compared to $Y$. enterocolitica, the effect of the process pressure on the inactivation is considerably smaller. Also, temperature between 5 and $40{ }^{\circ} \mathrm{C}$ does not seem to affect inactivation of $S$. aureus by highpressure homogenisation, while $Y$. enterocolitica inactivation is affected by temperature over a much wider range.

\subsection{Mathematical modelling}

In a first approach, we tried to fit the data with a complex second-order polynomial model (model 1). The model parameters and the adjusted $r^{2}$ and RMSE are given in Table 2, the analysis of the optimisation criteria in Table 3. Subsequently, one by one the
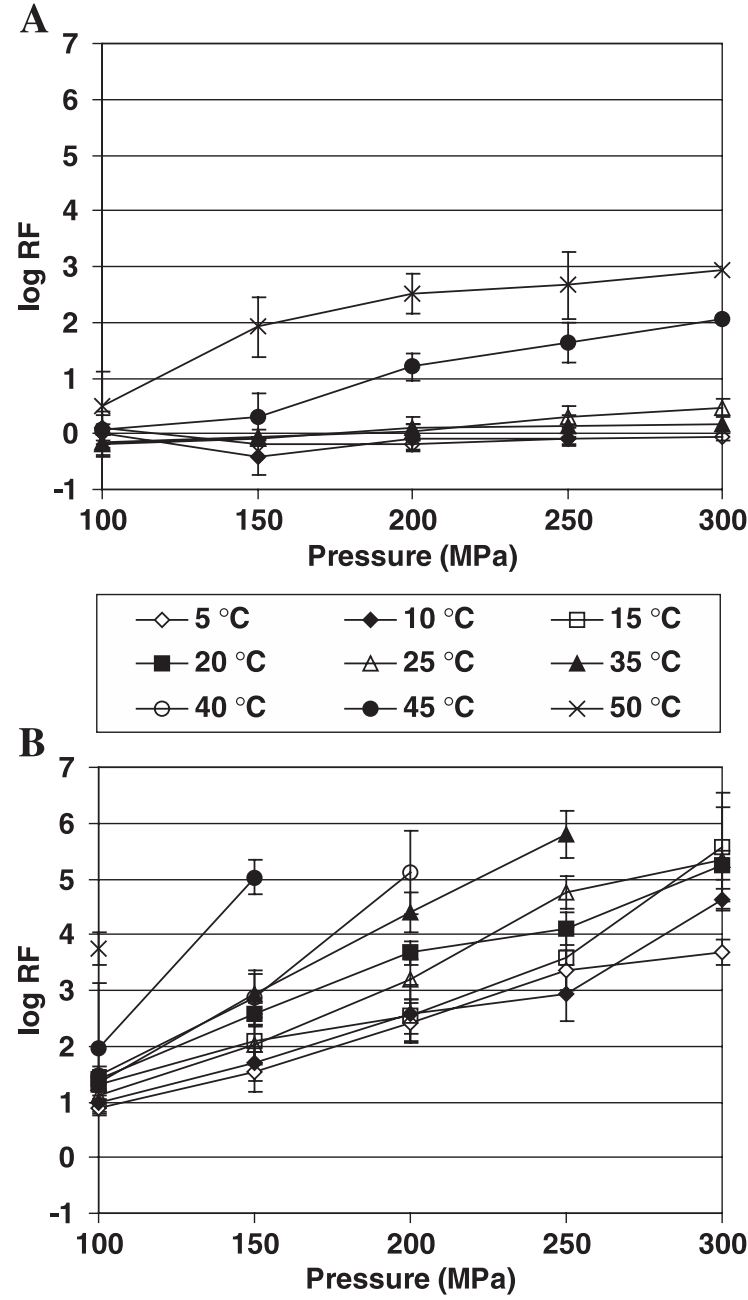

Fig. 1. Inactivation of S. aureus (A) and Y. enterocolitica (B) as a function of the homogenisation pressure at different process temperatures. $\log \mathrm{RF}=\log N_{\mathrm{o}} / N$. The presented data are the mean value of three experiments \pm standard deviation.

nonsignificant variables were removed by using the backward regression method until a model with only significant variables remained. This resulted for both $S$. aureus and $Y$. enterocolitica in the elimination of the quadratic pressure term (model 2, see Table 2). Elimination of other variables resulted in an increase of the $C_{p}$ criterion and RMSE and in a reduction of the adjusted $r^{2}$ (data not shown). However, for $Y$. enterocolitica, the estimation of the intercept $a$ was very bad $(t=-0.150)$, as can be seen from the high standard error. Therefore, we carried out a separate back- 
Table 2

Estimated parameters ( \pm standard error) of different models describing inactivation of $S$. aureus and $Y$. enterocolitica in PBS buffer as function of the process temperature $\left({ }^{\circ} \mathrm{C}\right)$ and pressure $(\mathrm{MPa})$

\begin{tabular}{|c|c|c|c|c|}
\hline \multirow[t]{2}{*}{ Parameter } & \multicolumn{4}{|l|}{ Estimated value } \\
\hline & Model 1 & Model 2 & Model 3 & Model 4 \\
\hline \multicolumn{5}{|l|}{ S. aureus } \\
\hline$a$ & $0.891 \pm 0.532$ & $1.03 \pm 0.335$ & & \\
\hline$b$ & $-0.130 \pm 0.016$ & $-0.130 \pm 0.016$ & $-0.115 \pm 0.015$ & $-0.0899 \pm 0.0111$ \\
\hline$c$ & $0.00204 \pm 0.00025$ & $0.00205 \pm 0.00024$ & $0.00193 \pm 0.00024$ & $0.00174 \pm 0.00024$ \\
\hline$d$ & $-0.0018 \pm 0.0050$ & $-0.00345 \pm 0.00153$ & $0.00584 \pm 0.00217$ & $0.000647 \pm 0.000791$ \\
\hline$e$ & $-4.21 \mathrm{e}-06 \pm 0.000012$ & & $-2.00 \mathrm{e}-04 \pm 7.86 \mathrm{e}-06$ & \\
\hline \multirow[t]{2}{*}{$f$} & $\begin{array}{l}0.00029 \pm 0.00005 \\
\text { adjusted } r^{2}=0.817\end{array}$ & $\begin{array}{l}0.000291 \pm 0.000045 \\
\text { adjusted } r^{2}=0.806\end{array}$ & $\begin{array}{l}0.000248 \pm 0.000039 \\
\text { adjusted } r^{2}=0.845\end{array}$ & $\begin{array}{l}0.000184 \pm 0.000030 \\
\text { adjusted } r^{2}=0.806\end{array}$ \\
\hline & $\mathrm{RMSE}=0.417$ & $\mathrm{RMSE}=0.414$ & $\mathrm{RMSE}=0.422$ & $\mathrm{RMSE}=0.438$ \\
\hline \multicolumn{5}{|c|}{ Y. enterocolitica } \\
\hline$a$ & $0.0956 \pm 0.6042$ & $-0.059 \pm 0.394$ & & \\
\hline$b$ & $-0.0825 \pm 0.0273$ & $-0.0819 \pm 0.0271$ & $-0.0799 \pm 0.0214$ & $-0.0855 \pm 0.0133$ \\
\hline$c$ & $0.0016 \pm 0.0004$ & $0.00160 \pm 0.00039$ & $0.00157 \pm 0.000357$ & $0.00164 \pm 0.000297$ \\
\hline$d$ & $0.0112 \pm 0.0054$ & $0.0130 \pm 0.0016$ & $0.0120 \pm 0.00244$ & $0.0127 \pm 0.000806$ \\
\hline$e$ & $4.29 \mathrm{e}-06 \pm 0.000013$ & & $2.77 \mathrm{e}-06 \pm 8.26 \mathrm{e}-06$ & \\
\hline \multirow[t]{2}{*}{$f$} & $\begin{array}{l}0.000039 \pm 0.000072 \\
\text { adjusted } r^{2}=0.870\end{array}$ & $\begin{array}{l}0.000386 \pm 7.10 \mathrm{e}-05 \\
\text { adjusted } r^{2}=0.870\end{array}$ & $\begin{array}{l}0.000383 \pm 0.000055 \\
\text { adjusted } r^{2}=0.972\end{array}$ & $\begin{array}{l}0.000395 \pm 4.10 \mathrm{e}-05 \\
\text { adjusted } r^{2}=0.973\end{array}$ \\
\hline & $\mathrm{RMSE}=0.587$ & $\mathrm{RMSE}=0.585$ & $\mathrm{RMSE}=0.585$ & $\mathrm{RMSE}=0.583$ \\
\hline
\end{tabular}

Model 1: $\log \mathrm{RF}=a+b T+c T^{2}+d P+e P^{2}+f T P ;$ model 2: $\log \mathrm{RF}=a+b T+c T^{2}+d P+f T P ;$ model $3: \log \mathrm{RF}=b T+c T^{2}+d P+e P^{2}+f T P ;$ model 4: $\log \mathrm{RF}=b T+c T^{2}+d P+f T P$.

ward regression on the model without an intercept. Application of the backward regression method showed in this case all parameters to be significant for S. aureus (model 3) $(P<0.1)$, and the further

Table 3

Optimisation criteria of different models describing inactivation of $S$. aureus and $Y$. enterocolitica in PBS buffer as function of the process temperature and pressure

\begin{tabular}{lcccc}
\hline $\begin{array}{l}\text { Optimisation } \\
\text { criteria }\end{array}$ & Model 1 & Model 2 & Model 3 & Model 4 \\
\hline S. aureus & & & & \\
AIC & -129 & -131 & -128 & -123 \\
SBC & -115 & -119 & -116 & -114 \\
PRESS & 14.3 & 14.0 & 14.6 & 15.6 \\
$C_{p}$ & 6.00 & 4.12 & 5.00 & 9.52 \\
& & & & \\
Y. enterocolitica & & & & \\
AIC & -137 & -139 & -138 & -141 \\
SBC & -120 & -124 & -124 & -129 \\
PRESS & 50.5 & 49.4 & 49.6 & 48.8 \\
$C_{p}$ & 6.00 & 4.11 & 5.00 & 3.11 \\
\hline
\end{tabular}

Model 1: $\log \mathrm{RF}=a+b T+c T^{2}+d P+e P^{2}+f T P ;$ model $2: \log \mathrm{RF}=$ $a+b T+c T^{2}+d P+f T P ;$ model $3: \log \mathrm{RF}=b T+c T^{2}+d P+$ $e P^{2}+f T P$; model $4: \log \mathrm{RF}=b T+c T^{2}+d P+f T P$. elimination of variables resulted in an increase of the $C_{p}$ criterion and RMSE and in a reduction of adjusted $r^{2}$ (data not shown). For $Y$. enterocolitica, the backward regression method applied to the model without an intercept resulted in the elimination of the quadratic pressure term (model 4). Elimination of additional variables resulted in an increase of the $C_{p}$ criterion and RMSE and in a reduction of adjusted $r^{2}$ (data not shown).

The residual plots and experimental-fitted plots for model 2 (S. aureus) and model 4 (Y. enterocolitica) are shown, respectively, in Figs. 2 and 3. The plots for the other models were similar. Based on the adjusted $r^{2}$ (see Table 2), RMSE (see Table 2), the residual plots and experimental-fitted plots, we can conclude that all of the proposed models fit well the data of $Y$. enterocolitica, but somewhat less so for $S$. aureus. Depending on the model, the adjusted $r^{2}$ is between 0.87 and 0.98 for $Y$. enterocolitica, and between 0.80 and 0.85 for $S$. aureus. On the other hand, based on the RMSE as a criterion, the fit is better for $S$. aureus (0.41-0.44) than for $Y$. enterocolitica (0.58-0.59). Furthermore, the experimental-fitted plots show no 

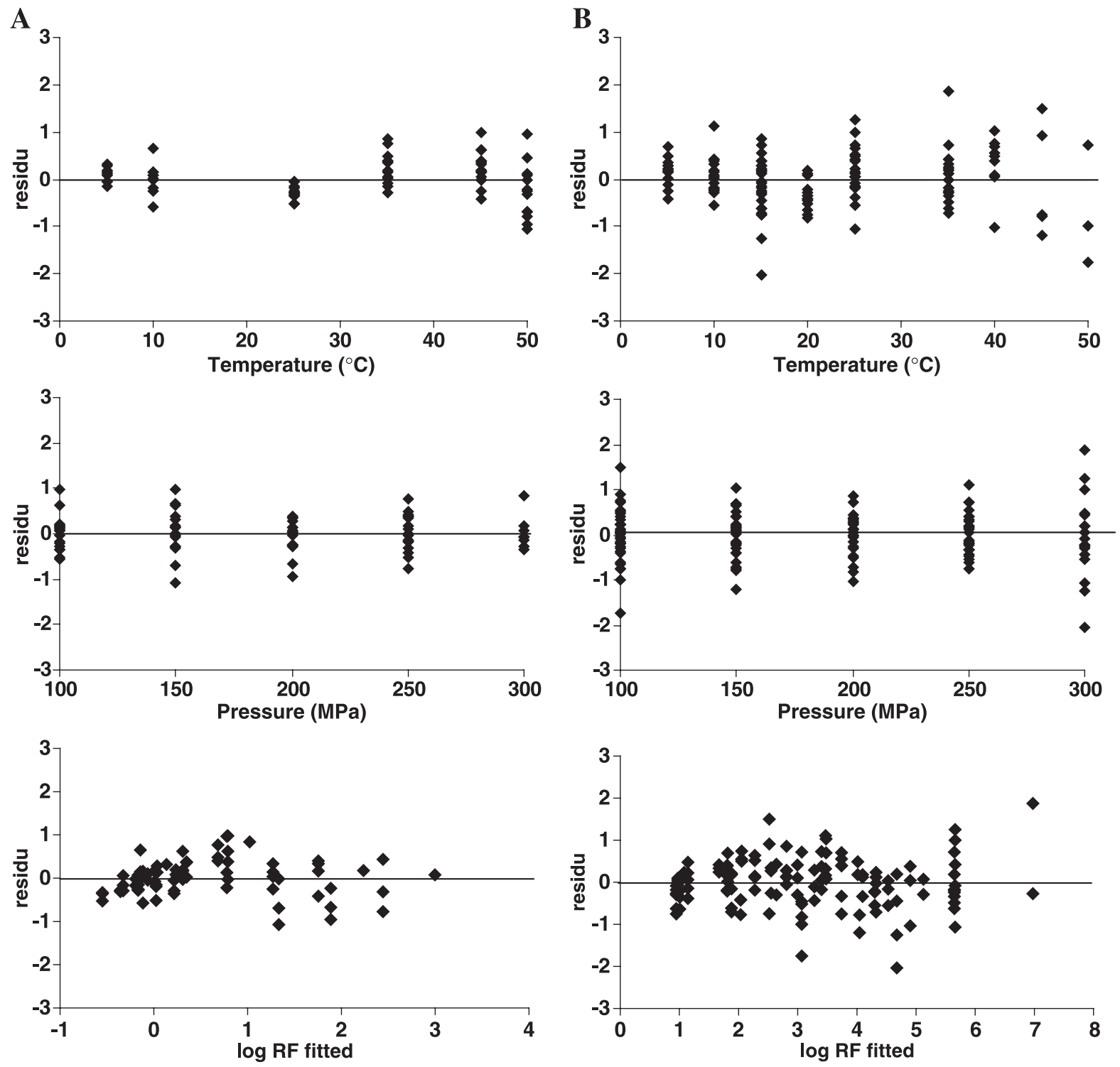

Fig. 2. Residual analysis of the inactivation of S. aureus (A) and Y. enterocolitica (B) according to, respectively, model 2 and model 4.

local over- or underestimation of $Y$. enterocolitica inactivation by the proposed models. For $S$. aureus in contrast, the analysis shows a slightly underestimated inactivation when the experimental $\log \mathrm{RF}$ is higher than 2. However, from a safety point of view, such an underestimation of inactivation is acceptable and certainly to be preferred above an overestimation. The residual plots show a random distribution for both $Y$. enterocolitica and S. aureus, except the plot of the residuals against the fitted $\log \mathrm{RF}$ for the latter organism, which shows a rather uneven distribution of the residuals around the zero line, probably due to the underestimation of the inactivation when the experimental log RF is higher than 2 (or the fitted $\log$ RF higher than 1.2).

For $Y$. enterocolitica, it can be concluded that model 4 results in the best fit (highest adjusted $r^{2}$ (0.973) and lowest RMSE (0.583)) and good estima- 

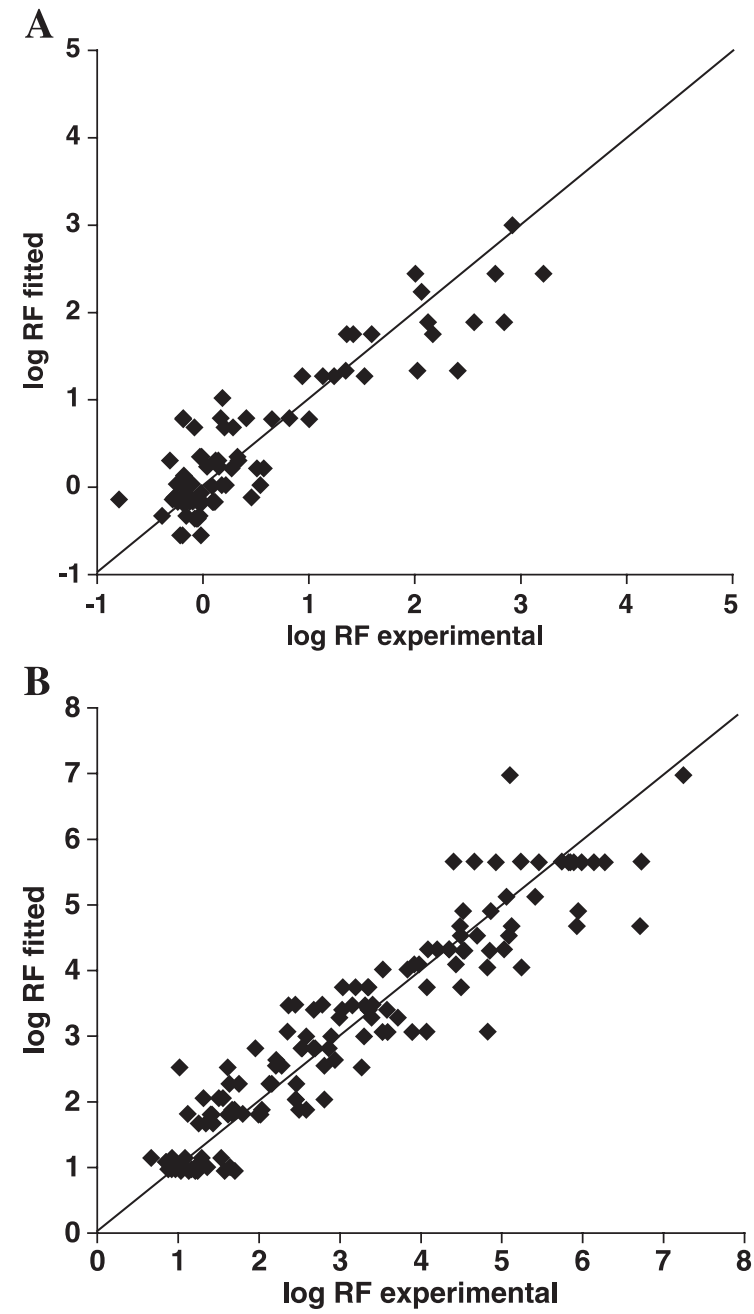

Fig. 3. Correlation between the experimentally determined log RF of $S$. aureus (A) and $Y$. enterocolitica (B) after high-pressure homogenisation and log RF calculated according to, respectively, model 2 and model $4 . \log \mathrm{RF}=\log N_{\mathrm{o}} / N$.

tion of all parameters $(|t|>5)$. For $S$. aureus, model 3 resulted in the highest adjusted $r^{2}(0.845)$, while model 2 resulted in the lowest RMSE (0.414). Both models resulted in fairly good parameter estimation $(|t|>2)$. As further criteria for model selection, we applied statistical comparison of the models by calculating AIC, SBC and PRESS of log RF. This comparison is valid because the errors of log RF can be assumed to be normally distributed. It appears from this analysis that model 2 and model 4 are most suitable, respectively, for $S$. aureus and $Y$. enterocoli- tica, since those models have the lowest $C_{p}$, AIC, SBC and PRESS (Table 3). Each of these individual criteria can be criticised, but since all give the same answer, the result is quite convincing.

A closer look on model 2 and 4 learns that the latter is a special case of the former in which the intercept is zero. Interestingly, when model 2 is applied to $Y$. enterocolitica, the estimated intercept is indeed very close to zero, and in fact, model 2 describes the data equally well as model 4 , however the addition of an extra parameter in model 2 is punished in our selection criteria since they take in account the number of parameters. Probably, since the intercept is so close to zero, it is difficult to accurately
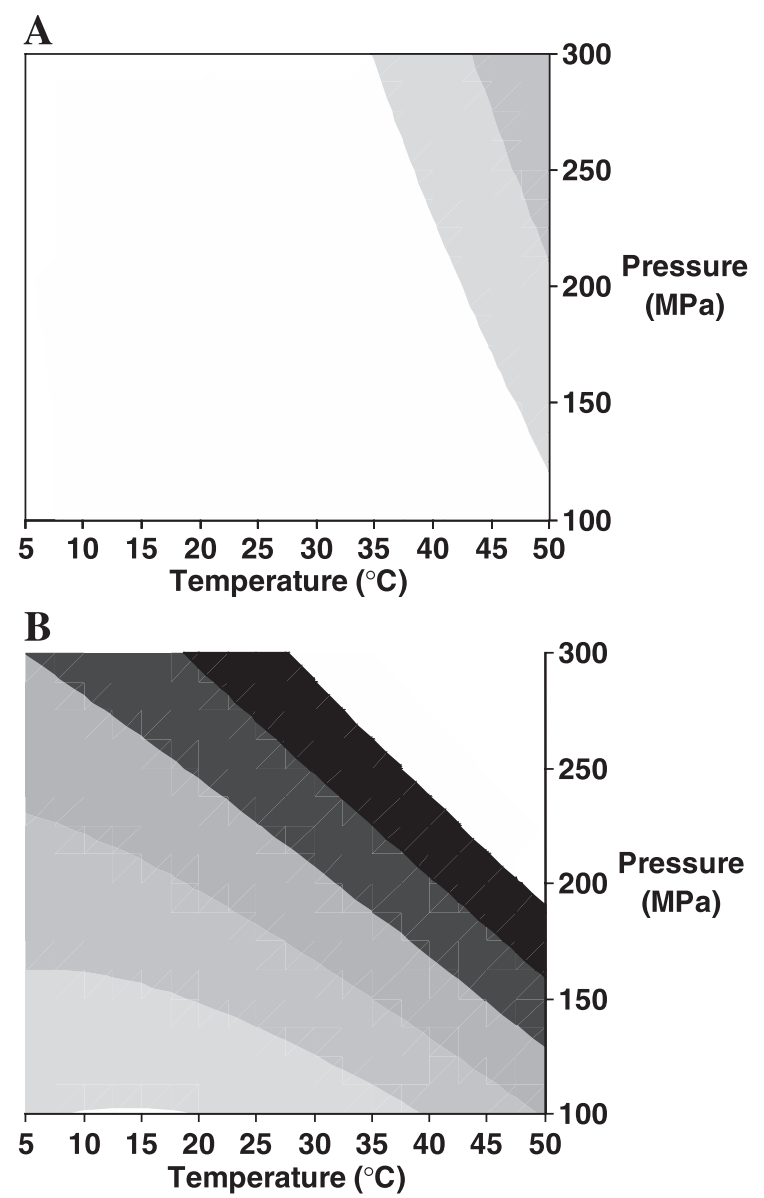

Fig. 4. Fitted iso-inactivation contour plot of S. aureus (A) and $Y$. enterocolitica (B) based on $\log \mathrm{RF}$ calculated, respectively, with model 2 and model $4 . \log$ RF between 1 and 2 ;2 and $3 \leftleftarrows ; 3$ and 4 ; 4 and $5 \square$; 5 and $6 \mathbf{\square}$. 
predict, which explains the high $p$-value in the $t$-test $(P=0.881)$. In conclusion, inactivation by high-pressure homogenisation can be described by model 2 as a common model structure for both bacteria. It will be interesting to see whether this model structure is also valid for other bacteria.

Based on $\log$ RF values calculated with the aid of model 2 for $S$. aureus and model 4 for $Y$. enterocolitica iso-log RF contours were determined. This is illustrated in Fig. 4A for S. aureus and in Fig. 4B for $Y$. enterocolitica.

\section{Acknowledgements}

E.W. has a postdoctoral grant from the Flemish Institute for the promotion of Scientific-Technological Research (I.W.T.), endorsed by Janssen Research Foundation (Beerse, Belgium). This work was supported by grants from the Belgian Fund for Scientific Research (F.W.O. G.0395.98) and K.U. Leuven Onderzoeksfonds (OT/97/31).

\section{References}

Akaike, H., 1969. Fitting autoregressive models for prediction. Ann. Inst. Stat. Math. 21, 243-247.

Doulah, M.S., Hammond, T.H., Brookman, J.S.G., 1975. A hydrodynamic mechanism for the disintegration of Saccharomyces cereviae in an industrial homogenizer. Biotechnol. Bioeng. 17, $845-858$.

Engler, C.R., Robinson, C.W., 1981. Disruption of Candida utilis cells in high pressure flow devices. Biotechnol. Bioeng. 23, $765-780$.
Guerzoni, M.E., Vannini, L., Chaves Lopez, C., Lanciotti, R., Suzzi, G., Gianotti, A., 1999. Effect of high pressure homogenisation on microbial and chemico-physical characteristics of goat cheeses. J. Dairy Sci. 82, 851-862.

Kelemen, M.V., Sharpe, J.E.E., 1979. Controlled cell disruption: a comparison of the forces required to disrupt different microorganisms. J. Cell Sci. 35, 431-441.

Keshavarz-Moore, E., Hoare, M., Dunnill, P., 1990. Disruption of baker's yeast in a high-pressure homogenizer: new evidence on mechanism. Enzyme Microb. Technol. 12, 764-770.

Lanciotti, R., Sinigaglia, M., Angelini, P., Guerzoni, M.E., 1994. Effects of homogenization pressure on the survival and growth of some food spoilage and pathogenic micro-organisms. Lett. Appl. Microbiol. 18, 319-322.

Lanciotti, R., Gardini, F., Sinigaglia, M., Guerzoni, M.E., 1996. Effects of growth conditions on the resistance of some pathogenic and spoilage species to pressure homogenization. Lett. Appl. Microbiol. 22, 165-168.

Mallows, C.L., 1973. Some comments on $C_{p}$. Technometrics 15, $661-675$.

Popper, L., Knorr, D., 1990. Applications of high-pressure homogenization for food preservation. Food Technol. 44, 84-89.

SAS online Doc, Version 8. http://mathstat.carleton.ca/ help/ sashtml/main.htm.

Save, S.S., Pandit, A.B., Joshi, J.B., 1994. Microbial cell disruption-role of cavitation. Chem. Eng. J. Biochem. Eng. J. 55, B67-B72.

Schwarz, G., 1978. Estimating the dimension of a model. Ann. Stat. 6, 461-464.

Shirgaonkar, I.Z.S., Lothe, R.R., Pandit, A.B., 1998. Comments on the mechanism of microbial cell disruption in high-pressure and high-speed devices. Biotechnol. Prog. 14, 657-660.

Vachon, J.F., Kheadr, E.E., Giasson, J., Paquin, P., Fliss, I., 2002. Inactivation of Foodborne pathogens in milk using dynamic high pressure. J. Food Prot. 65, 345-352.

Wuytack, E.Y., Diels, A.M.J., Michiels, C.W., 2002. Bacterial inactivation by high-pressure homogenisation and high hydrostatic pressure. Int. J. Food Microbiol. 77, 205-212. 